\title{
Decrease in Quadrivalent Frequency over a 10 Year Period in Autotetraploids in Two Species of Grain Amaranths ${ }^{1}$
}

\author{
M. Pal and R. M. Pandey \\ National Botanical Research Institute, Lucknow, India
}

Received August 11, 1981

Improvement in pollen and seed fertility has been observed in advanced generations of induced autotetraploids of a number of crop plants like maize (Gilles and Randolph 1951), Brassica campestris var. toria (Swaminathan and Sulba 1959), rye (Hilpert 1957), barley (Bender and Gaul 1966) and Coix (Venkateswarlu and Rao 1976). While in some cases the improvement in fertility could be correlated with reduced quadrivalent formation and increased bivalency (Hilpert 1957, Venkateswarlu and Rao 1976) in others no such correlation was evident (Muntzing 1951, McCollum 1958, Morrison and Rajathy 1960). With this in mind the situation was examined in two autotetraploid lines of $\mathrm{C}_{10}$ generation of two species of grain amaranths viz. Amaranthus caudatus Linn. and A. edulis Spegazzini of which the autotetraploids raised earlier ( $\mathrm{Pal}$ and Khoshoo 1977) were available for study. Analysis of these lines was made for their morphological features as also for pollen fertility. The cytological studies were done following usual technique (Darlington and LaCour 1976).

\section{Results}

Morphologically the plants of the $\mathrm{C}_{10}$ generation did not reveal any significant difference as compared to those reported for $C_{0}$ and $C_{1}$ generations and maintained the general gigantism of the determinate parts like leaves, stomata, pollen and seed. A small percentage of male plants in $\mathrm{C}_{1}$ as observed earlier by Pal and Khoshoo (1977) was also observed in $\mathrm{C}_{10}$ generation.

\section{Meiosis}

Both the species at the diploid level had $2 n=32$ and regularly formed 16 bivalents at meiotic metaphase $I$ followed by regular meiotic behaviour resulting in normal pollen fertility (for details see Pal and Khoshoo 1973 and 1977).

Meiosis in the $\mathrm{C}_{10}$ autotetraploids in the two species revealed varying number of quadrivalents, trivalents, bivalents and univalents at metaphase I (Figs. 1-4, Tables 1 and 2). The frequency of trivalents and the univalents, however, was very low (Tables 1-2). The mean frequencies of quadrivalents, trivalents, bivalents and univalents observed per cell were $2.31,0.66,25.93$ and 0.92 in $A$. caudatus and $1.91,0.67,26.76$ and 0.84 in $A$. edulis, respectively. On the whole, an average of less than 20 per cent chromosomes were involved in quadrivalent formation. It

1 NBRI Research Publication No. 134 (N.S.). 
appeared that the few trivalents and univalents, observed in low frequencies were actually disjoined quadrivalents while some univalents may be the result of precocious disjunction (Fig. 2). Cases of precocious disjunction have been reported

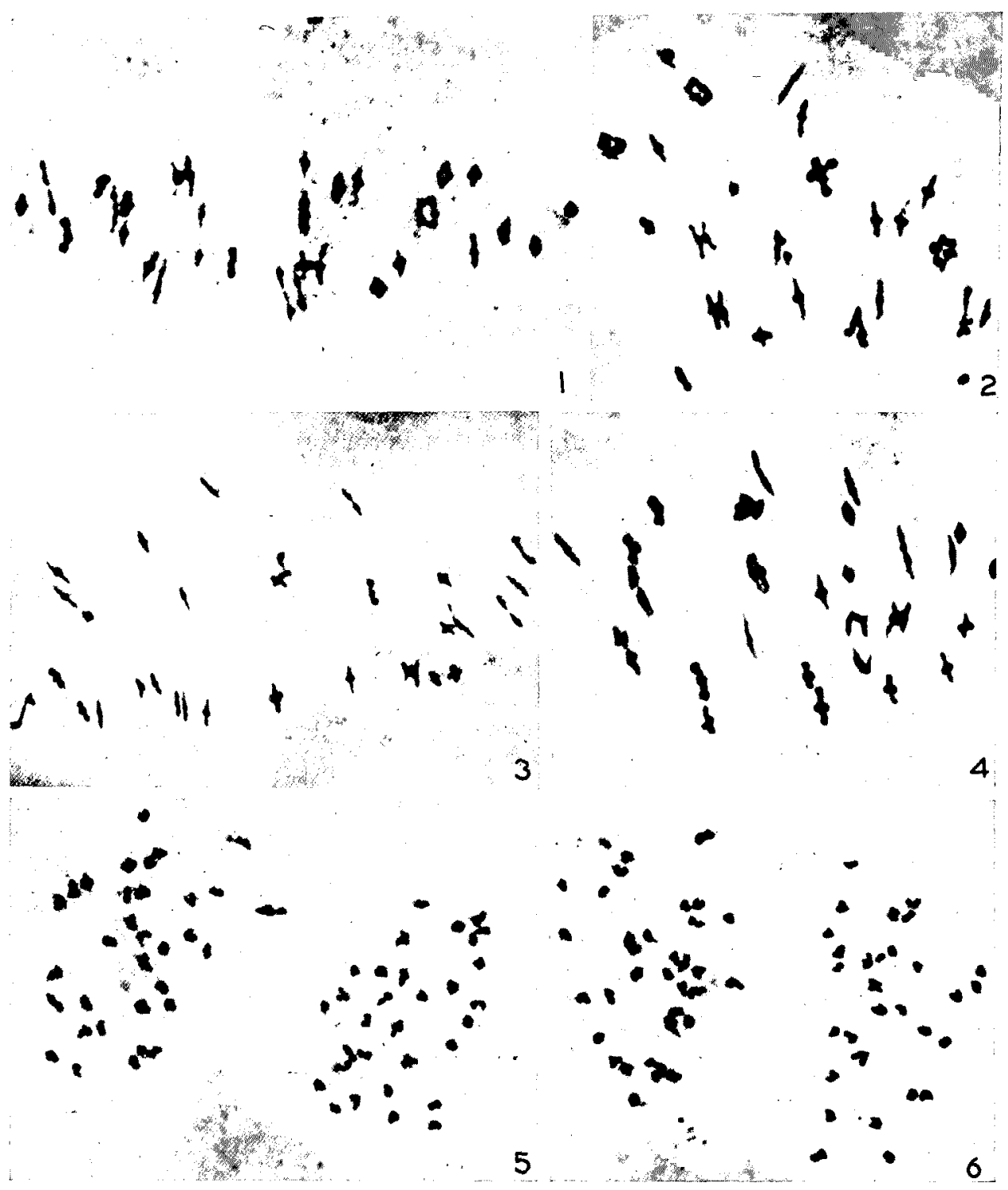

Figs. 1-6. 1 and 2, A. caudatus metaphase I, $3 \mathrm{IV}+26 \mathrm{II}$ and $8 \mathrm{IV}+1 \mathrm{III}+14 \mathrm{II}+1 \mathrm{I} . \quad 3$ and 4 A. edulis, metaphase $1,2 \mathrm{IV}+28 \mathrm{II}$ and $5 \mathrm{IV}+22 \mathrm{II}$. 5 and 6 , anaphase $1,32: 32 \mathrm{~A}$. caudatus, $A$. edulis. All $\times 1650$.

earlier in the diploids of the two species (Khoshoo and Pal 1972 and Pal and Khoshoo 1973).

Anaphase $I$ as a rule is regular in the autotetraploids in both the species resulting in 32: 32 distribution of chromosome (Figs. 5-6). However, a few cells 


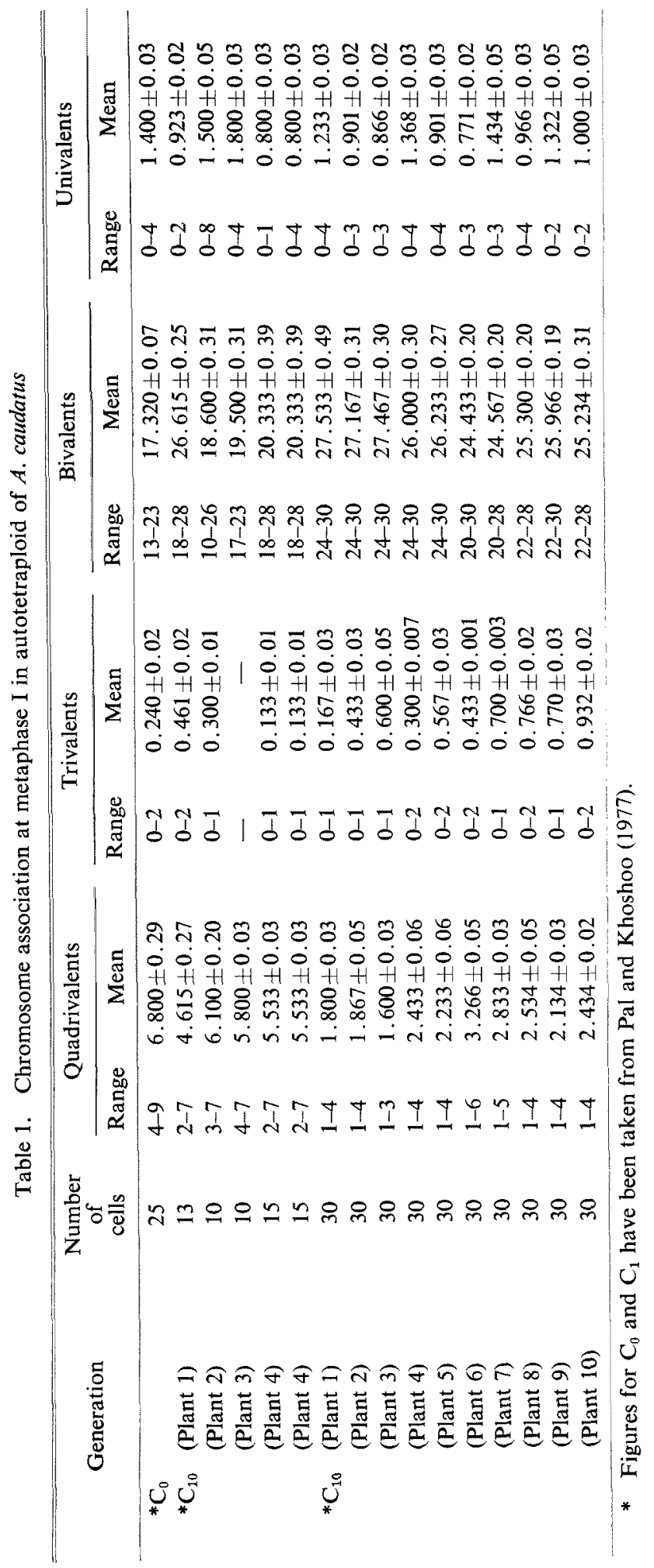




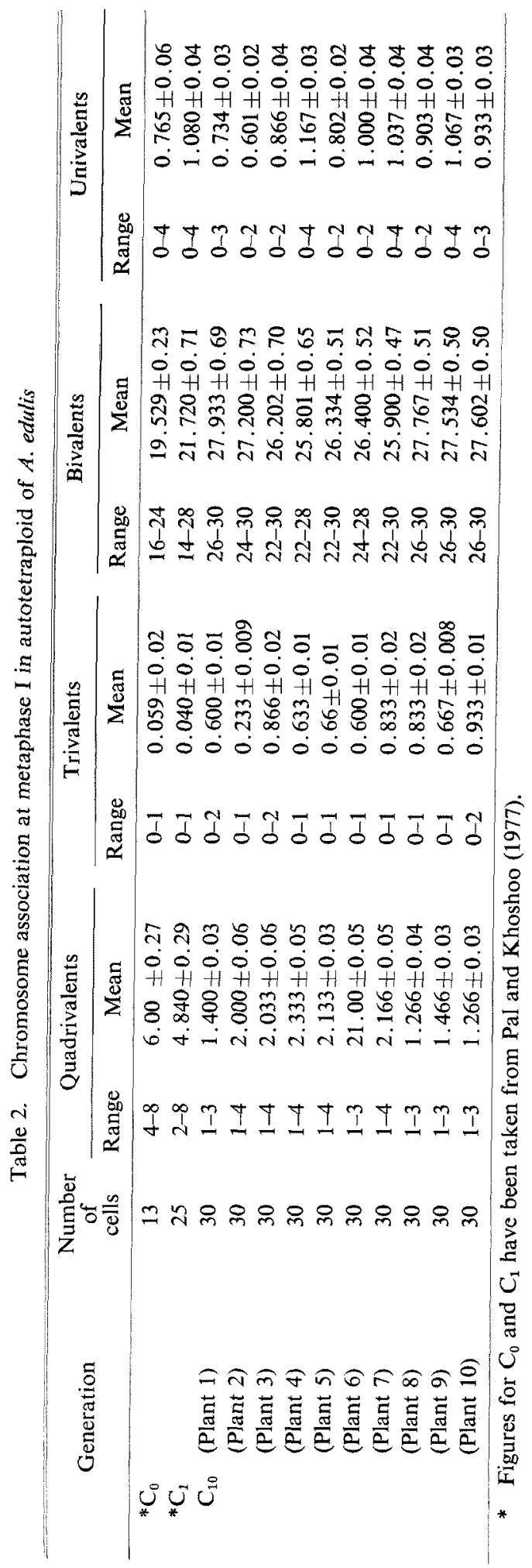


with laggards, bridges and unequal segregation and precocious division of univalents at anaphase I were also observed. These abnormalities perhaps resulted in the formation of micropollen which was recorded in low frequencies in the autotetraploid lines of both the species. Anaphase II was apparently normal.

\section{Discussion}

In contrast to the diploids the unique feature of the induced autotetraploids in both $A$. caudatus and $A$. edulis was the occurrence of stray predominantly male plants in the $\mathrm{C}_{10}$ generation. The occurrence of such predominantly male plants was also reported for these species in the $\mathrm{C}_{1}$ generation by Pal and Khoshoo (1977). Perhaps polyploidy disturbed the intricate genic balance for sex expression and brought about some sort of hormonal disturbance disrupting the normal and well set pattern of male and female flower formation. Such an abnormal development has also been reported for some of the interspecific hybrids in the genus (Pal 1972). It is therefore clear that the selection for ten generations has not resulted in the attainment of an entirely normal sex behaviour.

At metaphase I in the autotetraploid of the two species nearly $20 \%$ chromosomes were involved in quadrivalent formation. A few trivalents and univalents were also reported in these taxa for $\mathrm{C}_{0}$ and $\mathrm{C}_{1}$ generations ( $\mathrm{Pal}$ and Khoshoo 1977). The notable feature was the reduction in the frequency of quadrivalents. In the ten plants of $\mathrm{C}_{10} A$. caudatus which were studied the mean quadrivalent frequency varied from a minimum of $1.600 \pm 0.03$ to a maximum of $3.266 \pm 0.05$ (see Table 1). Similar reduction was noted in $\mathrm{C}_{10} \mathrm{~A}$. edulis also, where the minimum and maximum frequencies were $1.400 \pm 0.03$ and $2.33 \pm 0.05$, respectively (Table 2). The figures in $\mathrm{C}_{0}$ generations of mean quadrivalent frequency in the $A$. caudatus and $A$. edulis were $6.800 \pm 0.29$ and $6.00 \pm 0.27$, respectively. As is apparent these frequencies represent a considerable reduction when compared to the data for $\mathrm{C}_{0}$ and $\mathrm{C}_{1}$ generations in the respective species.

A similar decline in the quadrivalent frequency accompanying selection for fertility was also reported by Gilles and Randolph (1951) in tetraploid maize, Hilpert (1957) in rye, Swaminathan and Sulba (1959) in Brassica campestris var. toria, Bender and Gaul (1966) in barley and Venkateswarlu and Rao (1976) in Coix.

The reduction in quadrivalent frequency in the aforementioned cases appears to be correlated with inbreeding and selection of vigorous and fertile seed stock in the succeeding generations. The only possible inference which can be drawn from these observations is that the individuals with less quadrivalents and higher bivalent frequency were more fertile and vigorous. The increased bivalency perhaps resulted from increased preferential pairing through gradual differentiation and accumulation of heterozygosity over a number of generations.

It has been observed by Skirm (1942) and Sybenga (1966) that even small amount of structural heterozygosity leads to preferential pairing. Other reasons for the noted decrease in quadrivalent frequency could be the establishment of gene or gene complexes which suppressed multivalent association of chromosomes.

In contrast to these, instances of improvement in fertility associated with an 
increase in quadrivalent frequency have also been reported in rye (Müntzing 1951) and Dactylis (McCollum 1958). Morrison and Rajhathy (1960a) who studied a number of autotetraploids of different species found that there was neither evidence for heritable variation in quadrivalent formation nor for variation in the number of quadrivalents and their affect on fertility. They (Morrison and Rajhathy 1960b) also did not find any reduction in quadrivalent frequency in advanced generations of many autotetraploids studied by them. The data in the present study did not reveal any relationship between the formation of quadrivalents and univalents and other meiotic irregularities on the one hand, and the pollen sterility on the other. In the present investigations it was observed that the quadrivalent frequency did not affect the fertility of the plants. While Hazarika and Rees (1967) found a higher fertility correlated with higher quadrivalent frequency in rye. It may however be mentioned that the pollen and seed fertilities were high even in the $\mathrm{C}_{0}$ and $C_{1}$ generations of the present autotetraploids (see Pal and Khoshoo 1977). Moverover, in the isogenic autotetraploids as long as there is orderly disjunction of quadrivalents with no numerical chromosome differences, quadrivalent correlated sterility should not ordinarily be expected.

It is therefore obvious that whatever sterility was encountered may be more due to genetic physiological causes rather than irregular distribution of chromosomes on account of multivalent formation and other meiotic abnormalities.

However, whatever may be the reasons for improved fertility and the reduction in quadrivalent frequency, it is significant that in $C_{10}$ generation of the two induced autotetraploid lines of grain amaranths a considerable meiotic regularity has been achieved which, if the present studies are an indication is likely to improve further in the succeeding generations as a consequence of small chromosomes, low chiasma frequency, some amount of preferential pairing, high degree of cross pollination and heterozygosity and selection for vigour and fertility.

\section{Summary}

$\mathrm{C}_{10}$ autotetraploid lines in two grain species of Amaranthus viz. A. caudatus and $A$. edulis were evaluated for their morphology and meiosis. Morphologically the $\mathrm{C}_{10}$ plants in both the species maintained the general gigantism in the determinate parts like leaves, stomata, pollen and seed as observed in $\mathrm{C}_{0}$ and $\mathrm{C}_{1}$. The meiosis was typically autoploid with quadrivalents, trivalents, bivalents and univalents. The mean quadrivalent frequency as observed at metaphase I varied from a minimum of 1.600 to maximum of 3.266 in A. caudatus while the respective figures in $A$. edulis were 1.400 and 2.33. The mean quadrivalent frequencies for $\mathrm{C}_{0}$ generation in the two species were $6.800 \pm 0.29$ and $6.00 \pm 0.27$, respectively. These figures represented statistically significant reduction over $C_{0}$ and $C_{1}$ data. The present results have been discussed in relation to similar or contradictory observations of earlier authors on the metaphase I associations in advanced generations of autotetraploids. 


\section{Acknowledgements}

The authors are grateful to Dr. T. N. Khoshoo, Director, National Botanical Research Institute, Lucknow for guidance and encouragement and to Mr. T. K. Sharma and Mr. A. K. Sen Gupta for the illustrations.

\section{Literature cited}

Bender, K. and Gaul, H. 1966. Zur Frage der Diploidisierung autotetraploider Gerste. Z. Pflanzenzücht. 56: 164-183.

Darlington, C. D. and LaCour, L. F. 1976. Handling of Chromosomes. 6th Edn. George Allen \& Unwin Ltd. London.

Gilles, A. and Randolph, L. F. 1951. Reduction of quadrivalent frequency in autotetraploid maize during a period of ten years. Amer. J. Bot. 38: 12-17.

Hazarika, M. H. and Rees, H. 1967. Genotypic control of chromosome behaviour in rye. X. chromosome pairing in autotetraploids. Heredity 22: 317-332.

Hilpert, G. 1957. Effects of selection for meiotic behaviour in autotetraploid rye. Hereditas 43: 318-322.

Khoshoo, T. N. and Pal, M. 1972. Cytogenetic patterns in Amaranthus. Chromosomes Today 3: 259-267. Hafner Publ. Co., New York.

McCollum, G. D. 1958. Comparative studies of chromosomes pairing in natural and induced tetraploid. Chromosoma 9: 571-605.

Morrison, J. W. and Rajhathy, T. 1960a. Frequency of quadrivalents in autotetraploid plants. Nature 186: $528-530$.

- and - 1960b. Chromosome behaviour in autotetraploid cereals and grasses. Chromosoma (Berlin) 11: 297-309.

Müntzing, A. 1951. Cytogenetic properties and practical values of tetraploid rye. Hereditas 37: $18-84$.

Pal, M. and Khoshoo, T. N. 1973. Evolution and improvement of cultivated amaranths. VI. Cytogenetic relationships in grain types. Theor. Appl. Genet. 43: 242-251.

- and - 1977. Evolution and improvement of cultivated amaranths VIII. Induced autotetraploidy in grain types. Z. Pflanzenzuchtg. 78: 135-148.

Skirm, G. W. 1942. Bivalent pairing in an induced tetraploid of Tradescantia. Genetics 27: 635-740.

Swaminathan, M. S. and Sulba, K. 1959. Multivalent frequency and seed fertility in raw and evolved tetraploids of Brassica campestris var. toria. Z. Vererbungslehre 90: 385-392.

Sybenga, J. 1966. The role of meiotic chromosomic pairing pattern in induced structural allopolyploidization of autopolyploids. Acta. Agric. Scand. Suppl. 16: 43-45.

Venkateswarlu, J. and Rao, P. N. 1976. Effect of inbreeding and selection for vigour and fertility on meiotic behaviour in autotetraploid Job's Tears, Coix lacryma-jobi L. Theor. Appl. Genet. 47: 165-169. 